\title{
UNA ESPECIE NUEVA DE ARISTOLOCHIA L., SUBSECCIÓN PENTANDRAE (ARISTOLOCHIACEAE) DEL ESTADO DE COLIMA, MÉXICO
}

\author{
Francisco J. Santana-MicheL ${ }^{1}$ \\ Departamento de Ecología y Recursos Naturales \\ Centro Universitario de la Costa Sur \\ Universidad de Guadalajara \\ Apartado postal 64 \\ 48900 Autlán, Jalisco, México \\ Correo electrónico: fsantanam@cucsur.udg.mx
}

\begin{abstract}
RESUMEN
Aristolochia colimensis del estado de Colima, México, se describe como nueva especie. Se diferencia de los taxa relacionados de la subsección Pentandrae ( $A$. brevipes Benth., $A$. occidentalis Santana-Michel et $\mathrm{S}$. Lemus, $A$. oaxacana Eastwood y $A$. pringlei Rose) en el limbo del cáliz reflexo en la antesis y en su utrículo provisto de una giba en la parte superior.

Palabras clave: Aristolochia, Aristolochiaceae, Pentandrae, estado de Colima, México.
\end{abstract}

\begin{abstract}
Aristolochia colimensis is a new species described for the state of Colima, México. This species differs from related taxa of the subsection Pentandrae (A. brevipes Benth., A. occidentalis SantanaMichel et S. Lemus, A. oaxacana Eastwood and $A$. pringlei Rose) in its reflexed calyx limb at anthesis and in the utricle with a gibba on its upper part.

Key words: Aristolochia, Aristolochiaceae, Pentandrae, state of Colima, Mexico.

La sección Gymnolobus Duchartre agrupa a la mayoría de las aristoloquias mexicanas en la subsección Pentandrae Duchartre (Duchartre, 1854). Howard Pfeifer (1970), en su trabajo monográfico para las especies pentandras de Aristolochia L., reconoce para México 35, siendo su mayor aportación la descripción de 12 nuevas para la ciencia. En los últimos años se han hecho importantes contribuciones al conocimiento de esta subsección, como la descripción de cuatro especies adicionales (Santana-Michel, 1995; Ortega y Ortega, 1995; Santana-Michel y Lemus, 1996; Calzada et al., 1997). Las exploraciones botánicas intensivas en los estados de Jalisco y Colima han dado como resultado el descubrimiento de numerosas novedades para la ciencia, lo que indica que hace falta mucho trabajo por hacer referente a inventarios florísticos en todo el país.
\end{abstract}

\footnotetext{
${ }^{1}$ Estudiante de postgrado de la Facultad de Ciencias Biológicas y Agropecuarias, Universidad de
} Colima. 
En el presente trabajo se describe a Aristolochia colimensis, procedente de suelos calizos y bosque tropical caducifolio en el estado de Colima.

Aristolochia colimensis Santana-Michel sp. nov., Fig. 1.

Herbae perennes volubiles, 2-3 m longae; folia petiolata, attenuata vel acuminata, basi sagittata; flores axillares solitarii, calyx viridis, limbus reflexus ad anthesim, 11-15 mm longus, 5-7 mm latus, purpureus, fauce flavoviride, utriculus obconicus 6-nervis, gibbosus supra nervio medio, syringe excentrica, obconica, 2.5-2.75 $\mathrm{mm}$ longa.

Hierbas perennes, trepadoras, de 2-3 m de largo; raíz principal alargada, semileñosa, de $30-40 \mathrm{~cm}$ de largo; hojas pecioladas, ápice atenuado a ligeramente acuminado, base sagitada, haz y envés glabrescentes con pelos diseminados sobre las nervaduras principales y el borde, nervación actinódroma basal; pecíolo de (1.0) 1.8-2.3 (2.5) cm de largo, $1.5 \mathrm{~mm}$ de grueso; lámina de (4.0) 6-9 (11) cm de largo, 2-3 (4) cm de ancho; flores solitarias, axilares, pedúnculo bracteolado, de $4-8 \mathrm{~mm}$ de largo y $0.8-1 \mathrm{~mm}$ de grueso, bractéolas lanceolado-cordadas, pilosas, de 7-10 mm de largo, 5-9 mm de ancho; cáliz glabrescente-puberulento, el utrículo, el tubo y la parte abaxial del limbo de color verde claro, de 3-4 cm de largo, desarticulándose tres días después de la antesis; limbo, lanceoladoagudo, reflexo sólo durante la antesis, de 11-15 mm de largo, 5-7 mm de ancho, de color rojo purpúreo, superficie granular-punteada, los gránulos de $0.02 \mathrm{~mm}$, 5-nervado, las nervaduras principales evidentes, paralelas, las nervaduras perpendiculares inconspicuas; garganta verde amarillenta con máculas de color rojo purpúreo, pilosa, los pelos de 0.5$0.7 \mathrm{~mm}$ de largo; tubo ligeramente arqueado, de 10-17 mm de largo, 3-4 mm de diámetro; utrículo obcónico, 6-nervado, verde amarillento, de 7-9 mm de largo, 5-6 mm de ancho, piloso en la superficie interna, con una giba en la parte superior sobre la nervadura, siringe obcónica, excéntrica, de 2.5-2.75 mm de largo, diámetro inferior de 2-2.5 mm, diámetro superior de 3-3.5 mm, con las nervaduras bien marcadas; ginostemo 5-lobado, estipitado, de 3-3.75 mm de largo, $2.25 \mathrm{~mm}$ de diámetro, estípite de $1.5 \mathrm{~mm}$ de largo, estambres 5 , tetraloculares, de $2 \mathrm{~mm}$ de largo; ovario piloso, de 6-6.5 mm de largo, 1-1.5 $\mathrm{mm}$ de diámetro.

TIPO: México, Colima, municipio de Colima, La Salada, $17 \mathrm{~km}$ al sur de Colima, altitud $350 \mathrm{~m}$, bosque tropical caducifolio, 19 de noviembre de 1998, F. J. Santana-Michel y S. Lemus J. 9068 (holótipo en ZEA); isótipos por distribuirse a los herbarios ENCB, IBUG, IEB, y MEXU.

Distribución conocida: México, estado de Colima, solamente de la localidad tipo.

Época de floración: de octubre a noviembre y probablemente hasta diciembre.

Hábitat: Bosque tropical caducifolio en suelos calizos, pedregosos; asociada con las siguientes especies arbóreas: Gliricidia sepium (cacanahual), Gossypium aridum, Comocladia engleriana (hincha huevos), Cochlospermum vitifolium (panicua), Cnidoscolus spinosus (quemadora), Jacquinia macrocarpa subsp. pungens (asmol), Plumeria obtusa (cacaloxúchil), Cyrtocarpa procera (chupalcojote), Ceiba aesculifolia (pochote), Pseudobombax ellipticum 


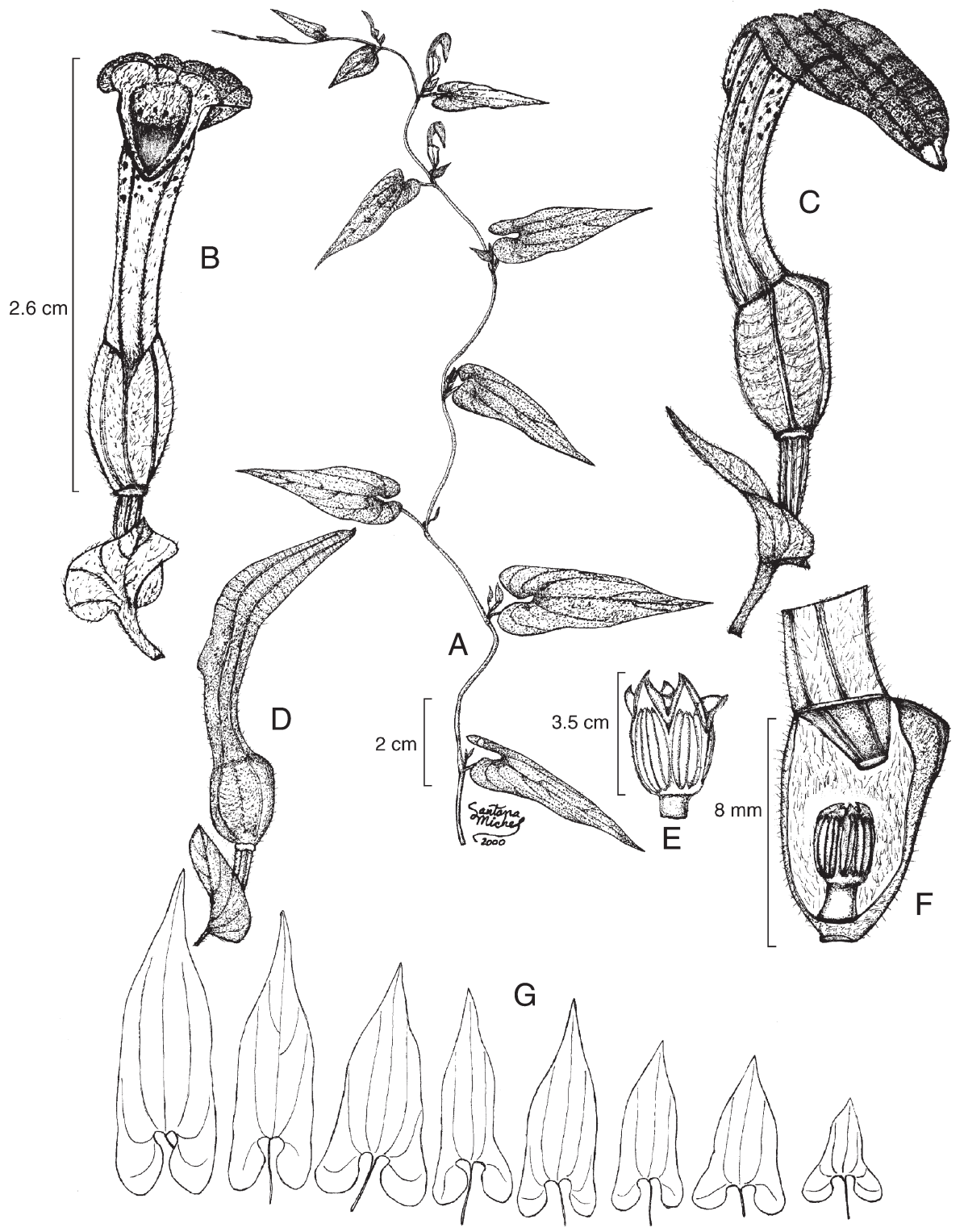

Fig. 1. Aristolochia colimensis. A. aspecto de la planta; B. flor vista de frente; C. flor vista de costado; D. flor cerrada; E. ginostemo inmaduro; F. corte longitudinal del utrículo, mostrando la siringe y el ginostemo maduro; G. variación de la hoja. Ilustración basada en la colección tipo ( $F$. J. Santana-Michel y S. Lemus J. 9068). 
(clavellina), Alvaradoa amorphoides (aretillo), Thouinia serrata (guayabillo), Cordia alliodora (hormigoso), Cordia elaeagnoides (barcino), Ziziphus mexicana (asmol), Amphipterygium adstringens (cuachalalate), Cedrela salvadorensis, Chomelia barbata, Neobuxbaumia squamulosa y especies arbustivas como Abutilon bastardioides, Mimosa brandegei, Senecio filaris, Hechtia laevis y Bromelia palmeri, entre las más abundantes.

Siguiendo la clave de Pfeifer (1970), Aristolochia colimensis se ubica junto a $A$. oaxacana Eastwood y $A$. brevipes Benth., en el grupo de las especies con los cálices maduros de 30-45 mm de largo y el tubo del cáliz subarqueado. El material vegetativo de A. colimensis puede confundirse con el de A. occidentalis Santana-Michel et S. Lemus y A. pringlei Rose. En el Cuadro 1 se resumen las diferencias entre todas estas especies.

Cuadro 1. Comparación de caracteres de A. colimensis con los de otras especies relacionadas.

\begin{tabular}{|c|c|c|c|c|c|}
\hline & A. occidentalis & A. pringlei & A. oaxacana & A. brevipes & A. colimensis \\
\hline Lámina foliar & $\begin{array}{l}\text { sagitado- } \\
\text { subcordada }\end{array}$ & $\begin{array}{l}\text { sagitado- } \\
\text { hastada }\end{array}$ & $\begin{array}{l}\text { ovada a } \\
\text { triangular }\end{array}$ & $\begin{array}{l}\text { triangular- } \\
\text { cordada }\end{array}$ & sagitada \\
\hline Largo del pecíolo & $1.3-4 \mathrm{~cm}$ & $3-4 \mathrm{~cm}$ & $3 \mathrm{~mm}$ & $2-3 \mathrm{~cm}$ & $1-2.5 \mathrm{~cm}$ \\
\hline Tamaño del cáliz & $5.5-6 \mathrm{~cm}$ & $6-11 \mathrm{~cm}$ & $3-3.5 \mathrm{~cm}$ & $3-3.5 \mathrm{~cm}$ & $3-4 \mathrm{~cm}$ \\
\hline $\begin{array}{l}\text { Limbo del cáliz } \\
\text { forma } \\
\text { postura } \\
\text { color }\end{array}$ & $\begin{array}{l}\text { lanceolado- } \\
\text { agudo } \\
\text { recto } \\
\text { verde claro }\end{array}$ & $\begin{array}{l}\text { lanceolado- } \\
\text { acuminado } \\
\text { recto } \\
\text { verde amari- } \\
\text { llento }\end{array}$ & $\begin{array}{l}\text { triangular- } \\
\text { agudo } \\
\text { recto } \\
\text { pardusco }\end{array}$ & $\begin{array}{l}\text { lanceolado- } \\
\text { obtuso } \\
\text { recto } \\
\text { café oscuro }\end{array}$ & $\begin{array}{l}\text { lanceolado- } \\
\text { agudo } \\
\text { reflexo } \\
\text { rojo purpúreo }\end{array}$ \\
\hline Utrículo & ovoide & ovoide & obcónico & obovado & $\begin{array}{l}\text { obcónico con } \\
\text { una giba }\end{array}$ \\
\hline
\end{tabular}

Las cinco especies en cuestión pueden separarse asimismo mediante la siguiente clave.

1 Limbo del cáliz 3 o más veces más largo que el tubo, cáliz de 5.5-11 cm de largo.

2 Cáliz de 6-11 cm de largo, limbo del cáliz lanceolado-acuminado A. pringlei

2 Cáliz de 5.5-6 cm de largo, limbo del cáliz lanceolado-agudo A. occidentalis

1 Limbo del cáliz menos de 3 veces más largo que el tubo, cáliz de $3-4.5 \mathrm{~cm}$ de largo.

3 Limbo del cáliz reflexo en la antesis, de color rojo purpúreo; utrículo con una giba en la parte superior A. colimensis

3 Limbo del cáliz recto en la antesis, de color café oscuro o pardusco; utrículo sin giba. 4 Hojas triangular-cordadas, pecioladas; bractéolas de 6-10 mm de largo; limbo del cáliz café oscuro, cubierto con pequeños tubérculos A. brevipes

4 Hojas ovado-triangulares, subsésiles o cortamente pecioladas; bractéolas de cerca de $1.5 \mathrm{~mm}$ de largo; limbo del cáliz pardusco, liso, sin tubérculos

A. oaxacana 
Aristolochia colimensis se puede identificar fácilmente por sus estructuras florales; el limbo es rojo purpúreo y reflexo (doblado hacia abajo), el utrículo obcónico con una giba en la parte superior sobre la nervadura, la garganta pilosa de color verde amarillento con máculas purpúreas.

\section{AGRADECIMIENTOS}

El autor desea agradecer a las autoridades del Centro Universitario de la Costa Sur de la Universidad de Guadalajara y especialmente al Departamento de Ecología y Recursos Naturales y a la Facultad de Ciencias Biológicas de la Universidad de Colima el apoyo en la realización de este trabajo. También se agradece al CONACyT la beca número 125840 . A Sebastián Lemus Juárez se dan las gracias por su ayuda en el trabajo de campo, a Roberto González Tamayo por la traducción de la diagnosis al latín, a los revisores anónimos por sus acertados comentarios y sugerencias a este trabajo, asimismo a mis compañeros y colaboradores en el inventario de la región Costa Sur de Jalisco Ramón Cuevas G., Luis Guzmán H., Nora Minerva Núñez L. y Enrique V. Sánchez Rodríguez.

\section{LITERATURA CITADA}

Calzada, J. I., G. Flores F. y O. Téllez V. 1997. Aristolochia davilae (Aristolochiaceae), a new species from Nayarit, Mexico. Contr. Univ. Michigan Herb. 21: 143-146.

Duchartre, P. 1854. Tentamen methodicae divisionis generis Aristolochia. Ann. Sci. Nat. Bot., sér. 4, 2: 29-76.

Ortega O., J. F. y R. V. Ortega O. 1995. Contribución al estudio de Aristolochiaceae de México, I. Una especie nueva de Aristolochia de Puebla, México. Acta Bot. Mex. 32: 39-45.

Pfeifer, H. W. 1970. A revision of the pentandrous species of Aristolochia. The University of Connecticut Publication Series. Storrs, Connecticut. $134 \mathrm{pp}$.

Santana-Michel, F. J. 1995. Una especie nueva de Aristolochia (subsección Pentandrae) del estado de Jalisco, México. Boletín IBUG 3(1-3): 87-90.

Santana-Michel, F. J. y S. Lemus J. 1996. Aristolochia occidentalis (Aristolochiaceae), una especie nueva de la subsección Pentandrae de Colima y Jalisco, México. Acta Bot. Mex. 36: 53-57. 